\title{
IMPLICACIONES DE LA DIVERSIDAD HUMANA Y FAMILIAR EN LA EVALUACIÓN DE CUSTODIA COMPARTIDA ${ }^{1}$
}

\author{
Jelitza Soto Román ${ }^{2}$
}

\section{Resumen}

La aprobación del Proyecto del Senado 63 a finales del año 2011 en Puerto Rico (Ley Protectora de los Derechos de los Menores en el Proceso de Adjudicación de Custodia) ha presentado toda una serie de retos para profesionales responsables en el proceso de evaluación, recomendación y adjudicación de custodia compartida. Reconociendo que la implantación de un proyecto de ley como el de custodia compartida representa, de manera muy particular, un reto para el/la profesional del Trabajo Social, resulta imperativa y necesaria una mirada reflexiva y de análisis crítico sobre el asunto. Es por tal razón que, en el siguiente trabajo se expone el análisis de la diversidad humana en términos de cultura, género, clase social, orientación sexual, religión, entre otras áreas, y sus implicaciones para el proceso de evaluación de custodia compartida. De igual forma, se abordan las múltiples complejidades que representan las diferencias de organización y estructura familiar en la evaluación de custodia compartida como una alternativa para el establecimiento de relaciones entre padres, madres y prole. Como profesionales de Trabajo Social, tenemos el compromiso de que nuestro desempeño y toma de decisiones refleje la ética y base valorativa de nuestra profesión. Más allá de una responsabilidad, es un deber y una obligación que nos

\footnotetext{
${ }^{1}$ Este artículo está basado en la ponencia presentada en la Octava Conferencia de Trabajo Social Forense celebrada en la Universidad del Turabo en Caguas, Puerto Rico, el 8 de agosto de 2013.

${ }^{2}$ Profesora a nivel subgraduado del Programa de Trabajo Social en la Universidad del Sagrado Corazón, San Juan, Puerto Rico.
} 
impone la autoridad profesional que nos ha sido conferida social y legalmente en el proceso de emitir recomendaciones para la toma de decisiones que afectarán las vidas de otras personas.

[Descriptores: custodia compartida, diversidad humana, diversidad familiar].

\section{Abstract}

The approval of the Senate's Project 63 at the end of the 2011 in Puerto Rico (Law for the Protection of the Minors' Rights during the process of Custody Adjudication) has represented a number of challenges for the professionals implicated in the joint custody evaluation, recommendation and adjudication process. Acknowledging that the implementation of a law project like this represents a challenge for Social Workers in a particular way, it is necessary and imperative a critical analysis about the topic. Fort that reason, the following article presents the analysis of human diversity in terms of culture, gender, class, sexual orientation, religion, among other areas, and their implications for the joint custody evaluation process. In the same way, it addresses the multiple complexities that represent the differences in family structure and organization in the joint custody evaluation as an alternative for the establishment of relations between parents and children. As social workers, we have the commitment to make sure that our professional performance reflects the ethics and core values of the profession. Beyond a responsibility, it is an obligation that our professional authority imposed to us in the process of making recommendations that will impact other people's lives.

[Keywords: joint custody, human diversity, family diversity].

\section{Introducción}

El siguiente artículo tiene como propósito analizar la diversidad humana en términos de cultura, género, clase social, orientación sexual, religión, entre otras dimensiones, y las implicaciones de estas en el proceso de evaluación de custodia compartida. De la misma manera, se abordarán las complejidades que representan las diferencias de organización y estructura familiar en la evaluación de custodia compartida como una alternativa para el establecimiento de relaciones entre padres, madres, y sus hijos e hijas. Se espera poder hacer una descripción general sobre cómo pueden incidir las distintas manifestaciones de la diversidad humana en los procesos de 
evaluación de custodia compartida. Además, se discutirá la importancia de considerar las diferencias en la organización y estructura familiar durante dichos procesos. Finalmente se abordará una reflexión crítica sobre las múltiples complejidades que representa la diversidad humana y familiar para la evaluación de custodia compartida. Para comenzar, debo mencionar que coincido con Nieves-Rolón (2009) en que analizar el tema de la custodia compartida es sumamente complejo debido a todas las partes y procesos involucrados, particularmente por sus implicaciones en las vidas y el desarrollo de los menores. Es por esto que, el trabajo estará dirigido a la importancia de tomar en cuenta aspectos de la diversidad humana y familiar en la evaluación de custodia compartida, de manera tal que el enfoque de las recomendaciones que surjan sea para el mejor bienestar de niños, niñas y adolescentes involucrados/as.

\section{Consideraciones de la diversidad humana en la custodia compartida}

Al hablar sobre custodia compartida resulta sumamente importante la consideración de elementos sobre la diversidad humana (características y diferencias distintivas según género, raza, religión, clase social, cultura, orientación sexual, edad, etc.); así como también, la identificación de fortalezas en las familias y sus miembros. Aunque la Ley 223 del 21 de noviembre de 2011 (Ley Protectora de los Derechos de los Menores en el Proceso de Adjudicación de Custodia) establece criterios específicos que el/la trabajador/a social debe tomar en consideración al hacer su evaluación, hay otros aspectos vinculados a la diversidad humana que también resultan importante considerar, o en los que resulta necesario hacer énfasis, durante dicho proceso. Para poder entender esto, es de particular importancia definir y clarificar el concepto de diversidad humana. Entonces, estaré partiendo de la siguiente definición del concepto: "diferencias que caracterizan a los seres humanos, las cuales tienen efectos en su conducta, su interpretación personal, autoimagen o identidad así como en las relaciones sociales que a su vez pueden resultar en discrimen y opresión hacia las personas o grupos" (Alegre, 2000, según citada en Feliciano \& López, 2012, p.23).

Uno de los principales aspectos sobre diversidad humana a ser considerado es el género. Cuando hago alusión al género estoy haciendo referencia a la forma en que se define socialmente lo que es ser hombre o mujer, utilizando como punto 
de partida el sexo biológico. Es una construcción cultural que implica el establecimiento de roles, funciones, responsabilidades, comportamiento y características (distintas) consideradas apropiadas para hombres y mujeres (Matos, 2008). Por ejemplo, en nuestra sociedad existe la creencia general de que el cuidado y la crianza de los hijos e hijas es una responsabilidad que le corresponde primordialmente a la mujer. Esta idea surge a partir de discursos vinculados al hecho de que por naturaleza es la mujer quien tiene la capacidad de dar a luz y amamantar. También, surge a raíz de discursos construidos desde un enfoque patriarcal donde se establecen relaciones desiguales entre hombres y mujeres. Como por ejemplo, aquel que establece que el hombre y la mujer pertenecen a esferas de actividad social distintas; el hombre a la esfera pública y la mujer a la esfera privada (Astelarra, 1984). El discurso que se genera, termina entonces apoyado en las subjetividades creadas por los propios hombres y mujeres, y validado así por la sociedad en lo referente al ejercicio de las tareas domésticas, la crianza de niños y niñas, y el cuidado de los demás; haciendo parecer como natural al género femenino en la crianza de la prole y la transmisión de emociones, mientras que al hombre se le adjudican otros tipos de funciones como ser proveedor económico (Nieves-Rolón, 2009).

Siendo personas que hemos crecido dentro de una sociedad patriarcal, este tipo de planteamientos seguramente forma parte de nuestras concepciones e ideas sobre lo que se supone debe hacer un hombre y una mujer en la ejecución de sus funciones de padre y madre respectivamente. Por ejemplo, uno de los criterios a considerar establecidos en la ley es la "capacidad de cada progenitor para satisfacer las necesidades afectivas, económicas y morales del menor" (Ley 223, 2011, Art. 7). Si dentro de nuestra concepción sobre los roles y capacidades que tienen los hombres y mujeres dentro de la crianza de los/as hijos/as entendemos que el componente afectivo corresponde a la mujer, podemos considerar que el padre del niño/a o adolescente no está capacitado para ello; lo cual podría influir en nuestro proceso de evaluación, inclinándonos a no recomendar la custodia compartida.

Como consecuencia de lo planteado anteriormente, surgen entonces las siguientes interrogantes: ¿a quién realmente beneficia el establecimiento o la asignación de esta función? ¿Al hombre, a la mujer misma, al hijo o hija? Seguramente 
las respuestas a dichas preguntas serán distintas según cada persona, porque las mismas partirán de las creencias y experiencias personales y culturales de cada quien. Sin embargo, al momento de considerar esto durante el proceso de evaluar la custodia compartida, resultará necesario desprenderse de dichas concepciones y narrativas. ¿Por qué? Porque estos discursos y subjetividades pueden permear nuestra manera de interpretar y analizar las distintas situaciones y circunstancias presentadas ante nosotros/as para su correspondiente evaluación. Esto podría implicar que las recomendaciones que se emitan sean a partir de dichos discursos y no necesariamente dirigidas hacia el mejor bienestar del menor.

Tal y como se plantea en la exposición de motivos de la Ley 223 del 21 de noviembre de 2011, es claro que los niños y niñas que son producto de hogares divorciados o de relaciones consensuales disueltas tienen las mismas necesidades que aquellos/as que están en hogares donde ambos progenitores están presentes. Sin embargo, también es importante reconocer que entre esas necesidades similares también existen diferencias que deben ser consideradas. Por ejemplo, cuando hablamos de diversidad funcional, tenemos que reconocer y considerar que aunque en nuestra sociedad el cuidado de los menores es asumido por las madres y los padres como una responsabilidad social y legal, dicha responsabilidad se convierte en un reto mayor cuando se trata de un hijo o hija con alguna discapacidad (Montañez, 2012). Esto debido a que este (a) requiere cuidados especializados y demanda mayor atención. Por tanto, es necesario considerar la condición, ya sea física o mental, del menor y conocer los cuidados y atenciones que requiere la misma. De esta forma, se estará en mejor posición de determinar si es un caso en el que la custodia compartida pudiera ser contraproducente para el bienestar de la niña o niño; o si por el contrario, favorecería el manejo de dicha condición por parte de sus progenitores.

Precisamente en el caso de los progenitores, un aspecto muy importante a considerar son las diferencias en cuanto a religión. En estos casos, las prácticas y creencias religiosas con las que se espera criar y enseñar al menor podrían representar un gran reto para el establecimiento de la custodia compartida. Particularmente cuando en los criterios a considerar establecidos en la ley se encuentra el nivel de "integridad moral exhibido por 
cada uno de los progenitores" (Ley 223, 2011, Art. 7, inciso 2). Generalmente, el sentido de la moral que tiene una persona tiende a estar intersecado por las ideologías religiosas, por lo que la interpretación de este concepto (integridad moral) podría variar de persona a persona. Es por tal razón que en este caso la concepción o sentido de moral que tenga la trabajadora o trabajador social resulta igualmente relevante, ya que podría permear el proceso de emitir recomendaciones en una dirección $u$ otra.

Lo anteriormente señalado también toma una relevancia o importancia particular cuando consideramos las diferencias en orientación sexual, ya que las concepciones o ideas que se tengan sobre esta; sea desde una visión religiosa o moral, podrían estar presentes en el proceso de evaluación de la custodia compartida. Un ejemplo que podría presentar al respecto es el hecho de que alguno de los progenitores, luego del divorcio o la terminación de la relación consensual, esté sosteniendo alguna relación de pareja con una persona de su mismo sexo (Toro-Alfonso, 2011). Otra posible circunstancia es que, el hijo o hija cuya custodia se está evaluando se encuentre manifestando tendencias homosexuales y el padre y madre tengan visiones diferentes al respecto. En cualquiera de los casos, ya sea que el aspecto de diversidad humana presente en la situación sea la religión o la orientación sexual, lo que debe dirigir nuestra evaluación y recomendación deberá ser nuevamente el mejor bienestar de los/as menores.

En esta misma línea, el considerar características de la diversidad humana que podrían posicionar en ventaja o desventaja (dinámicas de privilegio y opresión) al progenitor o progenitora, es otra área que resulta importante abordar en este trabajo. Particularmente la utilización de poder para coaccionar el interés o la disposición de uno/a u otro/a hacia la custodia compartida. Por ejemplo, cuando hablamos de progenitores de culturas diferentes, donde una de las culturas es considerada como superior a otra; cuando los niveles de educación entre el padre y la madre son distintos; y cuando la clase social a la que pertenecen es diferente. Cualquiera de estas circunstancias puede poner a alguno de los progenitores en una posición de poder o ventaja que le permita manipular o coaccionar a la otra parte para que cambie su parecer o acceda a la custodia compartida, por lo que como profesionales debemos prestar atención a esto. 
En este tipo de situaciones, si retomamos lo establecido en la ley sobre la "capacidad de cada progenitor para satisfacer las necesidades afectivas, económicas y morales del menor" (Ley 223, 2011, Art. 7, inciso 3), podría interpretarse que el hecho de que el padre o la madre pertenezca a una clase social baja no lo capacita económicamente para la crianza y el cuidado del hijo/a o los/as hijos/as. De igual forma, si señalamos la relación que generalmente se establece entre la clase social y el nivel de educación, también resultaría necesario considerar que el conocimiento que tiene una persona le otorga un poder que puede ser ejercido sobre otra cuya falta de conocimiento la ubica en una posición de vulnerabilidad. Por otra parte, cuando hablamos de cultura, un factor a considerar podría ser el estatus migratorio, ya que el mismo podría representar un elemento con el cual alguna de las partes esté presionando (coaccionando) a la otra para optar por la custodia compartida, independientemente esta sea beneficiosa o no para la menor o el menor.

Según lo plantean Feliciano y López (2012, p. 22), "cuando se actúa bajo prejuicio, se pone en ventaja o desventaja a un grupo con relación a otros, proveyendo oportunidades y privilegios a unos sectores considerados superiores 0 hegemónicos, mientras que para otras personas representan condiciones de inequidad, injusticias y discrimen". Por otro lado, también es necesario resaltar que el hecho de tomar en cuenta la clase social y el trasfondo cultural de alguno de los progenitores, o de ambos, implica adquirir o desarrollar una comprensión de dicho trasfondo: características de identidad, aspectos que integran los valores culturales y experiencias dentro del contexto cultural. De manera tal que podamos evaluar acertadamente el impacto (positivo o negativo) de esto en los procesos y realidades que se hacen presentes al compartir la custodia de cada menor.

Por último, un aspecto sumamente importante en la consideración de la diversidad humana durante el proceso de evaluar la custodia compartida, lo es la necesidad de informarse (revisión de literatura e investigaciones recientes) sobre el aspecto de diversidad humana identificado como significativo o relevante en cada situación. Así, estaremos en posición de hacer una evaluación y recomendación informada que le haga justicia a las partes involucradas, principalmente a las niñas o niños. En esta línea comparto nuevamente lo planteado por Nieves-Rolón (2009) al señalar que cada caso de custodia debe 
ser ponderado en sus méritos, ante iguales condiciones de los progenitores, siendo también iguales los deberes y derechos; ya que de esta forma y con una acción social concertada, habrá una mayor probabilidad de igualdad y justicia entre estos en beneficio del bienestar de los y las menores y de la sociedad en general.

\section{Diversidad familiar y su relación con la custodia compartida}

El abordaje de la diversidad familiar dentro del ejercicio de evaluar la custodia compartida resulta sumamente importante, pues a partir de procesos como este, las familias evolucionan y se adaptan. No obstante, según establece Martínez (2007),

No tenemos todavía en Puerto Rico un marco ideológico amplio o un macro sistema coherente y articulado, que recoja las transformaciones familiares desde una cultura de cambio y una perspectiva de respeto por la diversidad y la pluralidad. Ante esta ausencia se agudizan los prejuicios, la intolerancia, la desigualdad social, el desamparo y la exclusión de aquellas familias que no responden a la visión de la familia tradicional. (p. 168)

Un claro ejemplo de esto lo vemos en la correlación que se establece entre los hogares monoparentales y la delincuencia (aspecto al cual se hace alusión en la exposición de motivos de la ley), lo cual representa un prejuicio que se manifiesta en una generalización dirigida a que los hijos/as que crecen y son criados en familias monoparentales terminan siendo delincuentes. Aunque algunos estudios sobre delincuencia y criminalidad hechos en Puerto Rico coinciden en que la variable de hogares monoparentales (hogares principalmente a cargo de la madre) ha estado presente durante el crecimiento y crianza de personas involucradas en actividades delictivas; resulta necesario clarificar que otros aspectos como la violencia familiar (hacia los menores o entre la pareja), participación de otro miembro del núcleo familiar en actividades delictivas y situaciones económicas de pobreza, etc. también son factores que inciden en el hecho de que una persona se convierta o no en delincuente.

La alusión a la monoparentalidad como causa única o primordial de la delincuencia y criminalidad, es muestra clara de cómo la diversidad familiar es visualizada como algo negativo; dejando fuera del análisis variables adicionales como condiciones de vida, el acceso a recursos, situaciones de salud mental, entre otras. Este tipo de visión surge a partir de la tendencia que busca mantener el modelo de familia conyugal heterosexual como el 
modelo ideal y único saludable o adecuado para el bienestar del menor. Lo describe claramente Carballeda (2011) cuando plantea que,

...inclusive desde los imaginarios sociales la noción de hogar monoparental con 'cabeza' femenina o no, en muchos contextos se inscribe como una marca de disfuncionalidad, dispersión familiar, desintegración y como indicador de dificultades en el sostén económico y afectivo de los integrantes de la familia. (p. 167)

Otro ejemplo de la relación existente entre diversidad familiar y custodia compartida son precisamente las visiones que se tienen sobre la familia divorciada. Desde una visión psicológica se tiende a enfocar el divorcio como un proceso o situación caracterizada por conflicto y dolor para las personas (adultas y niños o niñas) implicadas. Sin embargo, desde una visión sociológica se reconoce como un proceso más complejo que trasciende el conflicto y sus efectos. Nos explica López-Cruz (2005) que:

Los estudios en torno a los efectos del divorcio de los progenitores en los niños y adultos no son concluyentes. Los más conservadores entienden que esa experiencia es devastadora o al menos nociva al bienestar de los niños y otros la ven como un reto, que aunque doloroso, a la larga trae crecimiento a todos los involucrados. Es posible que ambas opciones ocurran: el divorcio sea crítico y traumático para muchos y para otros, beneficioso al retar las capacidades y facilitar comportamientos novedosos para lidiar con el mismo. (p. 258)

La realidad es que en Puerto Rico coexisten una pluralidad de arreglos familiares que representan una gran complejidad que implica desigualdades y plantea nuevas situaciones que enfrentan las familias dentro de nuestra sociedad. Las expectativas sociales para las posiciones, roles y funciones de los miembros de la familia están confusas; ya que la normas que antes guiaban el comportamiento y las relaciones familiares están en proceso de revisión sin aún haber logrado un consenso (Albite, 2007). Esto lo vemos claramente ejemplificado en los retos que precisamente se están presentando en la implantación de la Ley 223, donde en el Artículo 3 se define la custodia compartida como:

...la obligación de ambos progenitores, padre y madre, de ejercer directa y totalmente todos los deberes y funciones que conlleva la crianza de los hijos, relacionándose con 
estos el mayor tiempo posible y brindándoles la compañía y atención que se espera de un progenitor responsable. (parr. 1)

Partiendo de esta definición, se observa que en la custodia compartida es necesario reconocer y distribuir las responsabilidades y obligaciones que bajo una custodia monoparental asume una sola de las partes (que en la mayoría de los casos es la mujer). No obstante, cabe destacar que para lograr esto se necesita una gran comunicación y buenas relacionas entre los excónyuges con el propósito de alcanzar arreglos para el bienestar emocional, físico e integral de sus hijos e hijas. Es necesario considerar cada contexto familiar y las circunstancias específicas que involucran a hombres, mujeres y niños o niñas; incluyendo sus posiciones dentro de la familia y las relaciones entre sus miembros. Por tanto, la observación y análisis de la dinámica familiar por parte de la trabajadora o trabajador social resulta sumamente importante; al igual que la consideración de diferencias en la organización y estructura familiar entre las distintas familias bajo su atención. En esta línea nos menciona Martínez (2007) que:

...desde el enfoque del construccionismo social podemos deconstruir y redefinir el género, la familia, el amor, los roles de padres y madres, padrastro y madrastra, el de los abuelos/as, las hermanas/os, los contratos matrimoniales, los vínculos, los compromisos, la vida cotidiana y tantos otros temas que condicionan emociones y conductas familiares. (p. 176)

Desde esta perspectiva se nos plantea como profesionales el reto de considerar la diversidad familiar dentro de la evaluación de custodia compartida, contribuyendo así al reconocimiento de la misma como algo válido y que puede ser beneficioso para los menores, siempre y cuando exista la disposición de las personas involucradas a trabajar para esto. Como plantea Felicitas (2011), "la diversidad de modelos familiares hace que la intervención con familias sea una y única en cada caso". (p. 31)

\section{Reflexiones para la práctica del Trabajo Social}

En el proceso de evaluar y considerar la custodia compartida se presenta la necesidad de retomar con mayor énfasis nuestra ética y valores profesionales, prestando atención de manera particular a que nuestros prejuicios y estereotipos no permeen la evaluación y análisis que estemos realizando. No 
podemos perder de perspectiva que el no estar conscientes de esto nos puede llevar a emitir recomendaciones sesgadas y seguramente poco justas para alguna de las personas implicadas. El prejuicio se define como una opinión, juicio, actitud o concepto formado sobre individuos, grupos o fenómenos basados en evidencia o pruebas no sistematizadas y no comprobadas en el carácter científico (Marsigilia \& Kulis, 2009, según citados por Feliciano \& López, 2012). También se define como una opinión, generalmente negativa, que se forma de antemano y sin el conocimiento necesario, lo que se traduce en actitudes hacia un grupo de personas por sus características sociales reales o imaginarias (Aguirre, 2007).

Es en el esfuerzo de reconocer esas ideas preconcebidas sin base cierta, que nuestra mirada profesional debe ir más allá de los prejuicios y enfocarse en el bienestar de las familias y su prole. Es una responsabilidad inescapable ejercer la profesión sin imponer nuestros valores y juicios personales, ya sea consciente o inconscientemente; y que toda acción y decisión que tomemos sea a partir de la ética profesional que nos dirige. Tenemos el compromiso de prepararnos adecuadamente para que nuestro desempeño como trabajadores y trabajadoras sociales refleje la base valorativa de nuestra profesión. Esta preparación implica un proceso constante de reflexión y autoevaluación que nos permita tomar decisiones que contemplen y respeten la diversidad humana y familiar en beneficio de las personas que servimos, particularmente de niños, niñas y adolescentes. Es más allá de una responsabilidad, un deber y una obligación que nos impone la autoridad profesional que nos ha sido conferida social y legalmente en el proceso de emitir recomendaciones para la toma de decisiones que afectarán las vidas de otras personas.

¿Cómo manejamos nuestros prejuicios y juicios sobre las personas y familias? ¿Estamos conscientes de ellos? ¿La consideración de tal o cual familia a la hora de la intervención profesional está regida por modelos teóricos, por nuestro propio modelo de familia o por un modelo ideal? Esta y otras preguntas son las que debemos hacernos al momento de realizar el análisis y evaluación correspondientes para someter nuestras recomendaciones. Como plantea Nieves-Rolón (2009), "cuando analicemos la situación debemos pensar en los procesos históricos que se han dado para estar donde estamos; concienciarnos del sesgo existente que de modo alguno favorecerá que la 
determinación sea una justa". (p. 306). Tenemos que reconocer que hemos sido socializados/as en la convergencia de diferentes discursos sobre la familia, por lo que ampliar las bases explicativas puede brindar solidez al análisis que realicemos. La realidad es que no hay una sola manera o forma de ser padres y madres; aunque culturalmente se haya definido y hayamos aprendido que es una la preferida o ideal. Por lo tanto, el uso del pensamiento crítico en el análisis y evaluación de la custodia compartida es fundamental y resulta imperativo para cada profesional de trabajo social. Carballeda (2011) reconoce esta realidad al mencionar que:

En el campo de la intervención en lo social, la relación con familias que clásicamente se denominan "inestables" es tal vez lo más frecuente. Esas nuevas formas de estructuración se presentan en el momento menos pensado, construyendo situaciones de incertidumbre, tanto en instituciones como en las prácticas que intervienen en éstas y en la aplicación de programas y políticas sociales. (p. 160)

Conscientes de que la implantación de un proyecto de ley como el de custodia compartida puede representar un reto para el/la profesional del Trabajo Social, resulta imperativo y necesario elaborar recomendaciones para hacer más efectivas las intervenciones; así como someter enmiendas que se entiendan necesarias a partir de las experiencias obtenidas en la práctica profesional. Además, resultaría necesario realizar estudios profundos sobre las diversas situaciones que se trabajan durante las evaluaciones de custodia compartida que permitan ampliar nuestro conocimiento sobre este asunto, y a su vez, desarrollar acciones acertadas en beneficio de los/as menores. Como bien señalan Feliciano y López (2012), la investigación social es otra vía de intervención y aportación del trabajo social en los procesos de transformación social, ya sea desde las narrativas de las personas o desde el análisis y medición de los fenómenos sociales.

A manera de conclusión, podemos establecer entonces que si analizamos la custodia compartida desde la pluralidad de características o ejes de la diversidad humana, estaremos en mejor posición de comprender la complejidad de las situaciones que nos sean referidas para evaluación. En cambio, es válido reconocer que la custodia compartida implica una forma de organización familiar que, más allá de no haber existido, está 
siendo reconocida por el Estado en el proceso de velar por el cumplimiento de las funciones que han sido asignadas a la familia (nuclear tradicional) dentro nuestra sociedad. Esto nos llevará a reconocer la importancia de nuestro rol en apoyar el desarrollo de nuevas dinámicas de relación, organización y estructuración familiar. Finalmente, no podemos olvidar que para enfrentar y minimizar las condiciones de opresión tenemos que transformar como profesionales nuestras actitudes, valores e ideologías que han sido construidas en el mismo contexto de las personas con las que trabajamos (Feliciano \& López, 2012). 


\section{Referencias}

Aguirre Esquer, Luis. (2007). Actitud y Diversidad Cultural. En Rosalie Soberal (Ed.). La Diversidad Cultural: Reflexión Crítica desde un acercamiento interdisciplinario (pp. 45-52). Mayagüez, Puerto Rico: Publicaciones Puertorriqueñas, Inc.

Albite Vélez, Lillian. (2007). La familia como una entidad socio-histórica: Implicaciones para la práctica de Trabajo Social. Revista Análisis, 8 (1), 45-73.

Astelarra, Judith. (1984). El feminismo como perspectiva teórica y como práctica política. República Dominicana: Ediciones de CIPAF.

Carballeda, Alfredo J.M. (2011). Algunos cambios en la esfera de la familia. Una mirada desde la intervención en lo social. En María Felicitas Elías (Compiladora). Nuevas formas familiares: Modelos, prácticas, registros (pp. 159-172). Buenos Aires, Argentina: Espacio Editorial.

Feliciano Giboyeaux, Isabel, \& López Ortiz Mabel T. (2012). Diversidad Humana y Dinámicas de la Opresión: Enfoque para el Trabajo Social. En Mabel T. López Ortiz (Ed.). Diversidad Humana: Retos y Compromisos del Trabajo Social (pp. 17-57). Rio Piedras, Puerto Rico: Publicaciones Puertorriqueñas.

Felicitas Elías, María. (2011). Las nuevas formas de familia y los desafíos del siglo XXI. En María Felicitas Elías (Compiladora). Nuevas formas familiares: Modelos, prácticas, registros (pp. 15-40). Buenos Aires, Argentina: Espacio Editorial.

Ley Protectora de los Derechos de los Menores en el Proceso de Adjudicación de Custodia, Ley

Núm. 223 del 21 de noviembre de 2011; Proyecto del Senado 63. San Juan: Estado Libre Asociado de Puerto Rico.

López-Cruz, Nilda. (2005) La familia: un enfoque interdisciplinario. San Juan, P.R. Publicaciones Lilo, Inc.

Martínez, Lourdes (2007). Familias y Exclusión: de la vergüenza al orgullo. En Rosalie Soberal, (Ed.). La Diversidad Cultural: Reflexión Crítica desde un acercamiento interdisciplinario (pp. 159-183). Mayagüez, Puerto Rico: Publicaciones Puertorriqueñas.

Matos, Mercedes. (2008). Las niñas sí silban: Afirmación personal y autoestima desde una perspectiva de género. Análisis, IX(1), 361-376.

Montañez Concepción, Isabel. (2012). Trabajo Social apoderando la persona discapacitada utilizando: Modelo de Solución de Problemas, perspectivas de fortaleza y sistémica. En Mabel T. López Ortiz (Ed.). Diversidad Humana: Retos y Compromisos del Trabajo Social (pp. 222-258). Rio Piedras, Puerto Rico: Publicaciones Puertorriqueñas. 
Nieves-Rolón, Irvyn. (2009). La custodia compartida: consideraciones desde la perspectiva de género masculino. En José ToroAlfonso (Ed.). Lo masculino en evidencia: investigaciones sobre la masculinidad (pp. 287-314). Hato Rey, Puerto Rico: Publicaciones Puertorriqueñas.

Toro-Alfonso, .2011). Hijo eres padre serás: La investigación sobre parentalidad de gays y lesbianas. En José Toro-Alfonso \& Alfonso Martínez-Taboas (Eds.). Lesbianas, gays, bisexuales y transgéneros: Apuntes sobre su salud desde la psicología (pp. 117-140). San Juan, Puerto Rico: Publicaciones Puertorriqueñas. 
